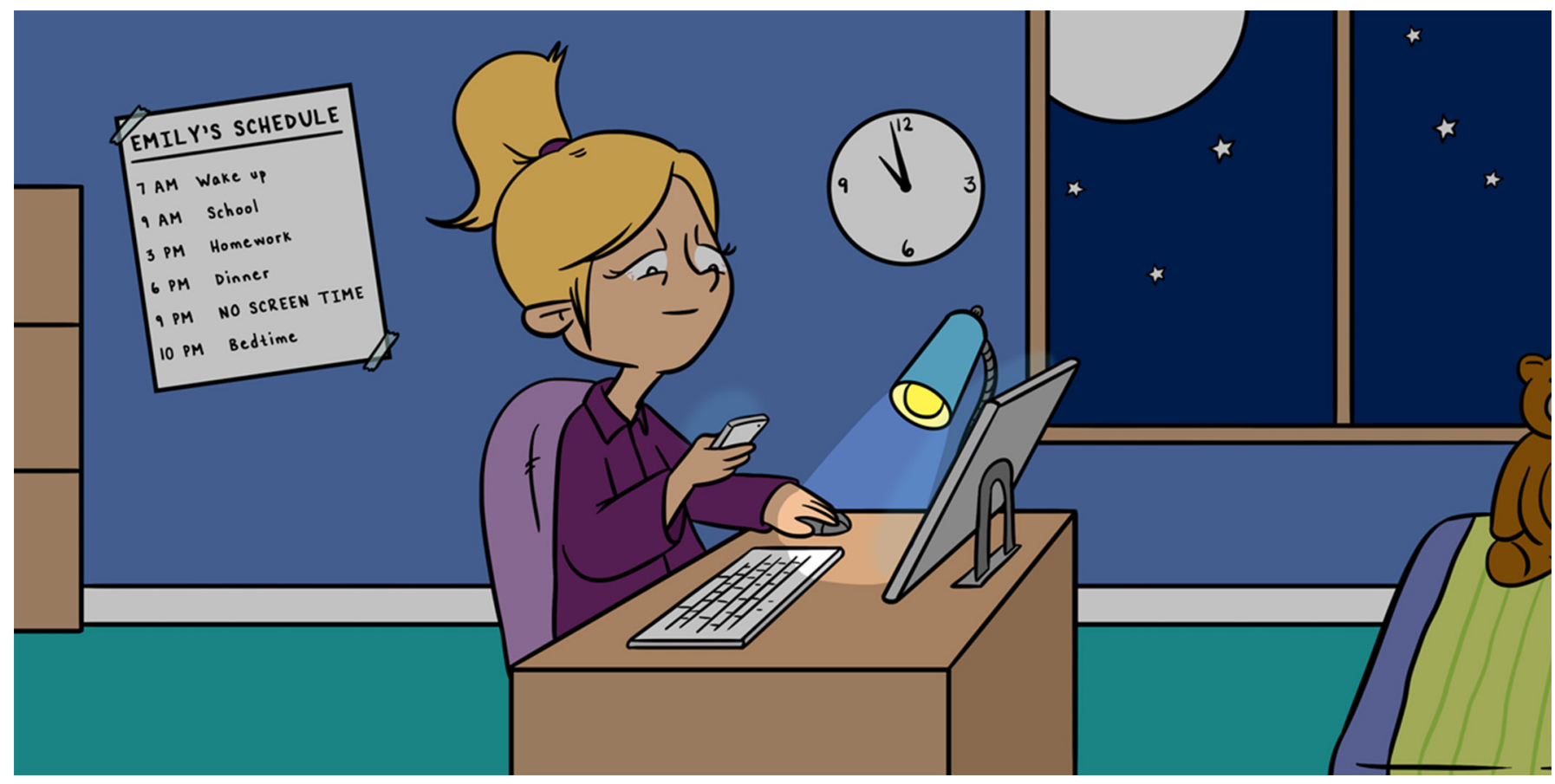

\title{
DAILY RHYTHMS OF THE BODY AND THE BIOLOGICAL CLOCK
}

\section{Tamar Shochat ${ }^{1}$ and Eran Tauber ${ }^{2 *}$}

${ }^{1}$ Department of Nursing, Faculty of Social Welfare \& Health Sciences, University of Haifa, Haifa, Israel

${ }^{2}$ Department of Evolutionary and Environmental Biology, Faculty of Natural Sciences, University of Haifa, Haifa, Israel

YOUNG REVIEWER:

ORI

AGE: 12
Earth's rotation creates a cycle of day and night, which is observed as changes in light levels and temperature. During evolution, plants and animals adapted to these cycles, developing daily cycles of physical and behavioral processes that are driven by a central biological clock, also known as the circadian clock. Even in the absence of changes in light between day and night, the biological clock creates cycles called circadian rhythms. The nervous system transfers information about the external light level to the biological clock in the brain, which matches the clock's cycle to the external environment. The biological clock prepares the body for environmental changes. The modern world has created disruptions in the circadian clock's timing, because of electrical lighting, flights to other time zones, and work during the night. The study of chronobiology studies the mechanisms of the biological clock and the clock's influence on human health. 


\section{INTRODUCTION}

Repeating processes, such as waves moving up and down, create a rhythm characterized by a consistent cycle. The earth's rotation creates the phenomenon of day and night in a 24-h cycle. This cycle results in environmental changes during the day, such as higher light levels and warmer temperatures. So, it is not surprising that, during evolution, the daily cycle was a significant factor to which animals and plants adapted. Many processes in our bodies show daily fluctuations, including our body temperature, blood pressure, and hormone levels. For example, the secretion of a hormone called melatonin (the sleep hormone) reaches its peak late at night and decreases in the morning, whereas, in the morning, the hormone cortisol reaches its peak. The concentrations of many other proteins in our bodies also show daily fluctuations. We are also all aware of daily cycles in our emotional and behavioral processes, such as our alertness and our ability to concentrate or learn, and the cycle of wakefulness and activity during the day and sleep during the night.

\section{THE BIOLOGICAL CLOCK CREATES DAILY RHYTHMS}

What drives the daily rhythms in our bodies? One possibility is that the body responds to cyclical changes in the environment. An increase in light levels with sunrise makes us wake up, and the darkness at night results in an increase in melatonin, which promotes sleep. An additional explanation is that there is an internal mechanism in the body, creating the daily rhythm independently of environmental changes. How can we differentiate between these two mechanisms? In 1962, a French scientist named Michel Siffre conducted an experiment on himself. He lived for 2 months in a cave in the Alps, without exposure to daylight, with constant temperature, and without knowing if it was day or night. He lived in a tent with an artificial lighting, connected to machines that tracked his body's activity. The results of the experiment (Figure 1) showed a daily, cyclical, organized activity pattern, where the significant difference was that each day he woke up about half an hour later. In fact, the length of the "day" he lived by in the cave was $24.5 \mathrm{~h}$. The main conclusion was that the body has an internal clock, which is independent of the environment's fluctuations. This clock creates daily rhythms, with a cycle slightly longer than $24 \mathrm{~h}$, even in the absence of environmental cues. In normal conditions, when we are exposed to sunlight, our clock cycle shortens to exactly $24 \mathrm{~h}$.

Sifrre's experiment earned him the nickname "the caveman," and received much publicity. But scientists who conducted similar experiments in animals and plants already knew about the existence of a biological clock and called it the circadian clock. In Greek, "circa" means "about" and "diem" means "day." The circadian clock exists in almost all animals on earth-in mammals, insects, plants, fungi, and 
Figure 1

Siffre's

cave experiment

The daily activity from the beginning of the experiment (top) to the end (bottom). The thick lines symbolize the time Siffre spent asleep. The first 10 days $(1-10)$ and last 10 days

(35-45) were spent in natural conditions outside the cave, and therefore his activity was synchronized to a 24-h clock. While in the cave, his activity reflects the cycle of the circadian clock, which is about $24.5 \mathrm{~h}$, which is why his sleeping time can be seen to shift to the right.

\section{PERIPHERAL}

\section{CLOCKS}

These clocks are in cells, tissues, and organs across the body. They receive information from the central clock in the brain and from other internal and external sources. For example, mealtimes are cues for peripheral clocks in the liver, kidney, and pancreas. The relationship between central and peripheral clocks is like the relationship between a conductor and musicians in an orchestra.

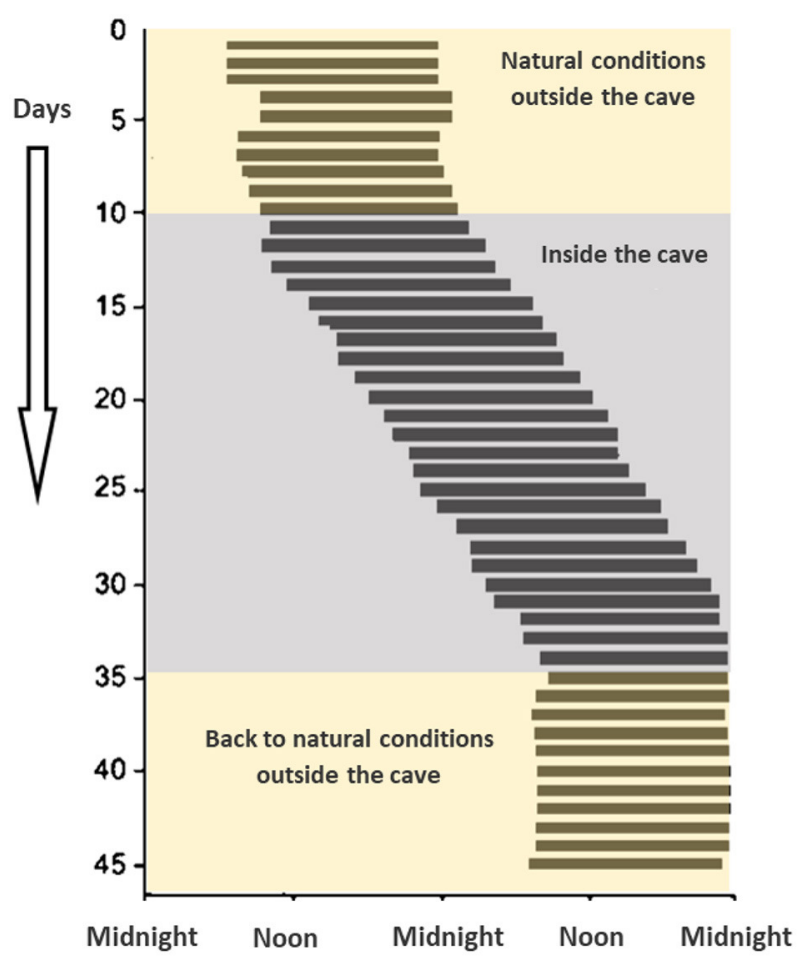

Figure 1

even bacteria. The cycle of the clock is different in different creatures, and even among individuals from the same species, and can be shorter or longer than $24 \mathrm{~h}$.

The ability to create daily rhythms exists in different cells, tissues, and organs in the body. These clocks, called peripheral clocks, are obedient to the central clock, which is located at the base of the brain in an area called the suprachiasmatic nucleus (SCN). This small region is the size of a rice grain and includes about 20,000 nerve cells. In experiments with mice in which the SCN is surgically removed, the behavior of the mice is quite normal, except that they lose the ability to keep their daily rhythms under constant conditions.

\section{WHY DO WE NEED THE CIRCADIAN CLOCK?}

We assume that the circadian clock exists in so many creatures because it provides some advantage to the survival of those species. To understand what this evolutionary advantage is, experiments were conducted on animals with faulty circadian clocks, both in the lab and the natural environment. In laboratory light-darkness conditions, animals without circadian clocks will still exhibit daily rhythms. For example, in lab conditions in which there is no danger of death from predators, fruit-flies with defective biological clocks have similar lifespan as flies with intact clocks. 
SUPRACHIASMATIC NUCLEUS (SCN)

A small brain structure that consists of around 20,000 nerve cells and function as the central clock. The SCN is located in the hypothalamus, above the area where the optic nerves from the eyes cross.

NEGATIVE

\section{FEEDBACK LOOP}

A process that paces itself. The thermostat is an example of negative feedback; when the temperature rises to a certain value, the thermostat turns off the heat. When the temperature goes down, the heating starts again. This process creates upward and downward temperature swings.

\section{SYNCHRONIZE}

Adjusting two waves to each other, so peaks and troughs coincide or present at a fixed time difference (synchronization is possible only between waves that have the same cycle length).
The advantage of the circadian clock becomes clear when examining animals in natural conditions, in competition with their own kind. For example, an experiment done on chipmunks demonstrated that, if the SCN was surgically removed, these rodents showed decreased survival in the forest, since their activity patterns were irregular and predators found them more easily [1]. Another experiment was conducted with a mix of two different species of bacteria, one with a short daily cycle $(23 \mathrm{~h})$ and the other with a long cycle $(30 \mathrm{~h})$ exposed to short and long days [2]. The results showed that one species pushed the other one aside. The "winning" species was the one whose circadian rhythm better suited the day's duration, so the species with the long cycle took over the culture in long-day conditions, while the species with the short cycle took over the culture that was grown in short-day conditions. These studies and others show that the circadian clock allows the body to act in harmony with the external environment.

\section{THE CLOCK'S GENETICS}

The first gene of the circadian clock was discovered in 1971, through research on a tiny fly called Drosophila, which is commonly used by genetic researchers [3]. The researchers gave the flies a chemical that damaged the genes in their DNA. Then they observed the daily activity of the flies and searched for those whose cyclical activity was disrupted. They soon found a fly in which the sleep and wakefulness times changed each day. This fly had lost its circadian rhythm because of one damaged gene, which got the name period (per for short). Per is a gene found in all animals, including humans. Later, the Clock gene was discovered (Clk for short) in mice. Over time, additional genes involved with the circadian clock were discovered.

The genes in animals are different from those in plants or fungi, but the principle of operation is similar, and is an example of a negative feedback loop. In this loop (Figure 2), the CLK protein stimulates the production of the PER protein. When the PER protein reaches a high level, it delays the production of the CLK protein. This delay results in a gradual reduction in the PER protein. The relationship between these two proteins leads to their daily fluctuations: when PER is high, CLK is low, and vice versa. These findings won three American scientists the Nobel Prize in physiology or medicine in 2017, for their studies using Drosophila.

\section{THE INFLUENCE OF LIGHT ON THE BIOLOGICAL CLOCK}

As Siffre discovered in his cave experiment, the circadian clock operates even without exposure to cycles of light and darkness, although the daily cycles are longer than $24 \mathrm{~h}$. In a natural environment, natural light is a cue that synchronizes us to a $24-\mathrm{h}$ clock. Additional time clues include temperature and social time cues, 
Figure 2

The molecular circuit of the clock. Activator proteins (such as CLK) in the cell nucleus increase the production of clock proteins, which are inhibitory (such as PER) The inhibitory proteins move back to the nucleus where they inhibit the activator proteins, slowing down their own production. When the level of the inhibitory proteins goes down, the inhibition stops and a new cycle resumes. This is an example of a negative feedback loop.

\section{INTRINSICALLY}

PHOTOSENSITIVE

RETINAL GANGLION CELLS (IPRGC)

A type of nerve cells in the retinas of mammals, which contain light receptors that do not participate in the process of seeing, but rather in the synchronizing of the circadian clock to light from the environment.

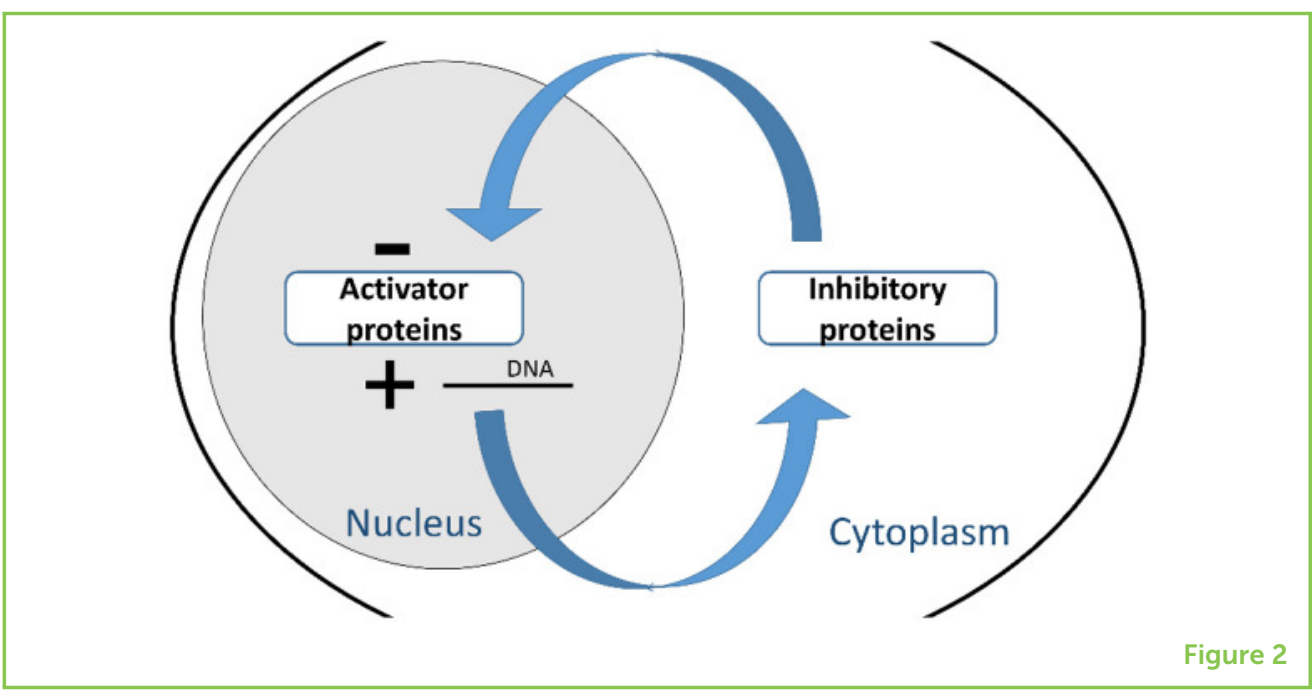

such as mealtimes, work times, and study times. But light is considered the strongest time cue influencing the circadian clock.

How does this happen? In the retina of the eye, there are three types of molecules that transform light energy to the electrical activity that travels to the brain. Two types of receptors, the rods and cones, are used for seeing. A third, lesser-known group of receptors is called intrinsically photosensitive retinal ganglion cells (ipRGCs). These receptors supply information to the nerve cells that connect the retina to the SCN. Two decades ago, researchers found that the ipRGCs contain a light-sensitive pigment called melanopsin, and they figured out how melanopsin helps to synchronize the circadian rhythms with the external environment [4].

\section{THE INFLUENCE OF MODERN LIFE ON THE BIOLOGICAL CLOCK}

Shortly before the identification of melanopsin, researchers discovered that the light-detecting molecules that influence the circadian clock are sensitive to blue light, which is light with a wavelength of 460 nanometers. This finding has great significance for the modern world, because today we are exposed not only to sunlight, but to electronic light sources that we use long after sunset. LED bulbs and devices, such as televisions, computers, and smartphones all produce blue light, therefore influencing our circadian rhythms.

There are other ways that modern life makes it harder for our circadian clocks to synchronize with the environment. A common example is flying to far-away countries, in which case the circadian clock must be delayed or brought forward by a few hours in order to synchronize. This process can take a few days and it is experienced as unpleasant sensations called jet lag. Jet lag can be caused by shifting the light cycle by just a few hours (Figure 3). Studies show that people frequently 


\section{Figure 3}

Jet lag experiment. An example of the activity of a hamster (nocturnal animal). The gray background symbolizes hours of darkness, and the yellow background symbolizes hours of light. After 8 days (top) the light is switched off $6 \mathrm{~h}$ earlier (indicated by the arrow). The hamster needs 1 week to synchronize to the new rhythm. The $6-h$ shift in the

light-darkness cycle is equivalent to the jet lag experienced after flying east, for example from Israel to Japan, where bedtime comes earlier.

\section{CHRONOBIOLOGY}

The scientific discipline that studies biological timing systems and their effects on health and functioning.

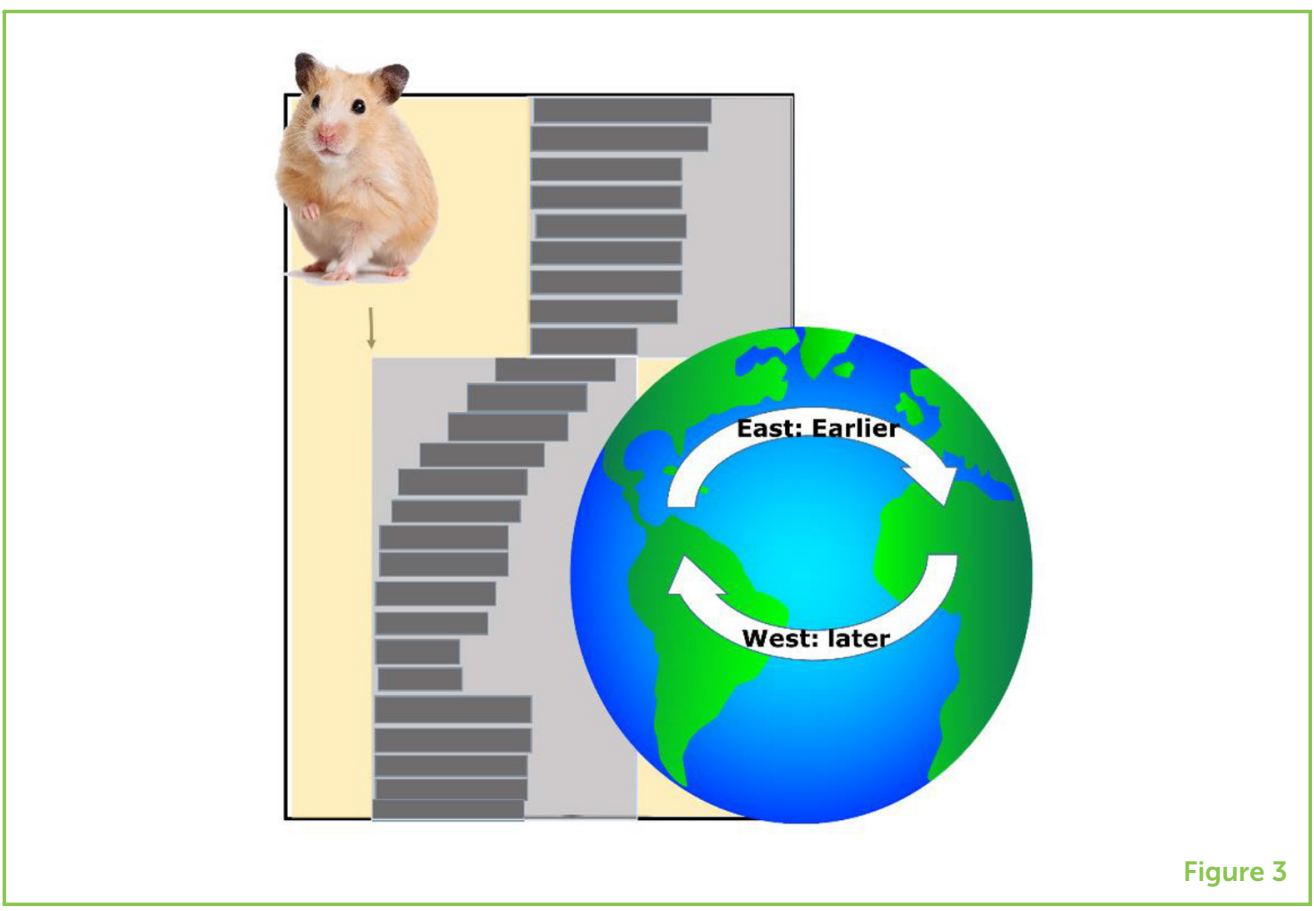

experiencing jet lag are at higher risk of cancer and other chronic illnesses. Higher levels of certain diseases/disorders are also seen in people who work at night, who are effectively experiencing prolonged social jet lag. To reduce the health damage that can result from these disruptions to the circadian rhythm, it is recommended that we are exposed to daylight as much as possible, and that we avoid the light from our screens at night.

\section{SUMMARY}

The circadian clock is built from genes and nerve cells that allow us to be in complete coordination with the daily fluctuations of the environment. Exposure to artificial light makes it harder for the circadian clock to synchronize with the environment, which could lead to health issues. The science of chronobiology (biological clock research) focuses on understanding the circadian clock's mechanisms and the differences in clocks between people. This knowledge will help scientists to develop personalized medicine in the future, which will consider the unique body rhythms of each and every one of us.

\section{REFERENCES}

1. DeCoursey, P. J., Walker, J. K., and Smith, S. A. 2000. A circadian pacemaker in free-living chipmunks: essential for survival? J. Comp. Physiol. A 186:169-80. doi: 10.1007/s003590050017 
2. Ouyang, Y., Andersson, C. R., Kondo, T., Golden, S. S., and Johnson, C. H. 1998. Resonating circadian clocks enhance fitness in cyanobacteria. Proc. Natl. Acad. Sci. U.S.A. 95:8660-4. doi: 10.1073/pnas.95.15.8660

3. Konopka, R., and Benzer, S. 1971. Clock mutants of Drosophila melanogaster. Proc. Natl. Acad. Sci. U.S.A. 68:2112-6. doi: 10.1073/pnas.68.9.2112

4. Gooley, J. J., Lu, J., Chou, T. C., Scammell, T. E., and Saper, C. B. 2001. Melanopsin in cells of origin of the retinohypothalamic tract. Nat. Neurosci. 4:1165. doi: $10.1038 / \mathrm{nn} 768$

SUBMITTED: 23 December 2020; ACCEPTED: 11 March 2021; PUBLISHED ONLINE: 20 April 2021.

EDITED BY: Idan Segev, Hebrew University of Jerusalem, Israel

CITATION: Shochat T and Tauber E (2021) Daily Rhythms of the Body and the Biological Clock. Front. Young Minds 9:645707. doi: 10.3389/frym.2021.645707

CONFLICT OF INTEREST: The authors declare that the research was conducted in the absence of any commercial or financial relationships that could be construed as a potential conflict of interest.

COPYRIGHT @ 2021 Shochat and Tauber. This is an open-access article distributed under the terms of the Creative Commons Attribution License (CC BY). The use, distribution or reproduction in other forums is permitted, provided the original author(s) and the copyright owner(s) are credited and that the original publication in this journal is cited, in accordance with accepted academic practice. No use, distribution or reproduction is permitted which does not comply with these terms.

\section{YOUNG REVIEWER}

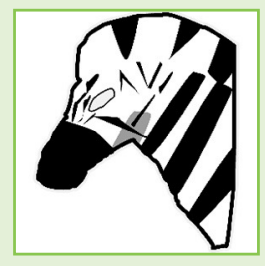

\section{ORI, AGE: 12}

My name is Ori and my favorite food is chopped Gruffalo. I spend most of my time at school. I like to program games and websites, design logos, edit videos, play the piano, and play on the computer. I participate in the program for talented youth in mathematics at Bar-Ilan University.

\section{AUTHORS}

\section{TAMAR SHOCHAT}

Tamar Shochat studies factors involved in sleep regulation as well as health and functional outcomes associated with human sleep. She studies mostly non-clinical populations that are particularly vulnerable to sleep disruption, such as adolescents, older adults, and shift workers, in their ecological surroundings. She is particularly interested in studying the consequences of mistimed sleep due to the conflict between the circadian clock and social constraints, also known as circadian misalignment or social jet-lag. This conflict may be due to evening light exposure, delayed sleep phase (evening types), and shift work. 


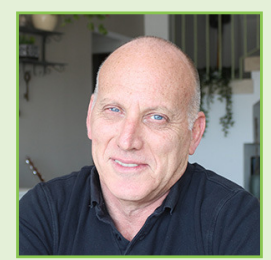

\section{ERAN TAUBER}

I am a scientist interested in the genetics of biological clocks and how changes in these genes allow the clock to function under different environmental conditions. We are also researching what genetic basis causes animals to be active at different times of the day. I head the laboratory for biological clocks at the University of Haifa and our main research animal is the fruit fly. *etauber@univ.haifa.ac.il 